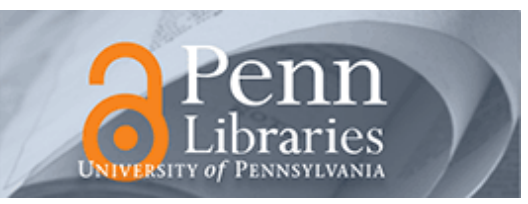

University of Pennsylvania

ScholarlyCommons

\title{
Mining Terabytes of Submillimeter-resolution ECoG Datasets for Neurophysiologic Biomarkers
}

\author{
Jonathan Viventi \\ University of Pennsylvania, jviventi@seas.upenn.edu \\ Justin Blanco \\ University of Pennsylvania, blancoj@seas.upenn.edu \\ Brian Litt \\ University of Pennsylvania, littb@mail.med.upenn.edu
}

Follow this and additional works at: https://repository.upenn.edu/be_papers

Part of the Biomedical Engineering and Bioengineering Commons

\section{Recommended Citation}

Viventi, J., Blanco, J., \& Litt, B. (2010). Mining Terabytes of Submillimeter-resolution ECoG Datasets for Neurophysiologic Biomarkers. Retrieved from https://repository.upenn.edu/be_papers/167

Suggested Citation:

Viventi, J., J. Blanco and B. Litt (2010). "Mining terabytes of submillimeter-resolution ECoG datasets for neurophysiologic biomarkers." Proceedings of the 32nd Annual International Conference of the IEEE EMBS. Buenos Aires, Argentina. August 31 - September 4, 2010.

(C2010 IEEE. Personal use of this material is permitted. However, permission to reprint/republish this material for advertising or promotional purposes or for creating new collective works for resale or redistribution to servers or lists, or to reuse any copyrighted component of this work in other works must be obtained from the IEEE.

This paper is posted at ScholarlyCommons. https://repository.upenn.edu/be_papers/167

For more information, please contact repository@pobox.upenn.edu. 


\title{
Mining Terabytes of Submillimeter-resolution ECoG Datasets for Neurophysiologic Biomarkers
}

\author{
Abstract \\ Recent research in brain-machine interfaces and devices to treat neurological disease indicate that \\ important network activity exists at temporal and spatial scales beyond the resolution of existing \\ implantable devices. We present innovations in both hardware and software that allow sampling and \\ interpretation of data from brain networks from hundreds or thousands of sensors at submillimeter \\ resolution. These innovations consist of novel flexible, active electrode arrays and unsupervised \\ algorithms for detecting and classifying neurophysiologic biomarkers, specifically high frequency \\ oscillations. We propose these innovations as the foundation for a new generation of closed loop \\ diagnostic and therapeutic medical devices, and brain-machine interfaces.

\section{Disciplines} \\ Biomedical Engineering and Bioengineering | Engineering

\section{Comments} \\ Suggested Citation: \\ Viventi, J., J. Blanco and B. Litt (2010). "Mining terabytes of submillimeter-resolution ECoG datasets for \\ neurophysiologic biomarkers." Proceedings of the 32nd Annual International Conference of the IEEE \\ EMBS. Buenos Aires, Argentina. August 31 - September 4, 2010. \\ (C)2010 IEEE. Personal use of this material is permitted. However, permission to reprint/republish this \\ material for advertising or promotional purposes or for creating new collective works for resale or \\ redistribution to servers or lists, or to reuse any copyrighted component of this work in other works must \\ be obtained from the IEEE.
}




\title{
Mining terabytes of submillimeter-resolution ECoG datasets for neurophysiologic biomarkers
}

\author{
Jonathan Viventi, Justin Blanco, and Brian Litt, Member, IEEE
}

\begin{abstract}
Recent research in brain-machine interfaces and devices to treat neurological disease indicate that important network activity exists at temporal and spatial scales beyond the resolution of existing implantable devices. We present innovations in both hardware and software that allow sampling and interpretation of data from brain networks from hundreds or thousands of sensors at submillimeter resolution. These innovations consist of novel flexible, active electrode arrays and unsupervised algorithms for detecting and classifying neurophysiologic biomarkers, specifically high frequency oscillations. We propose these innovations as the foundation for a new generation of closed loop diagnostic and therapeutic medical devices, and brain-machine interfaces.
\end{abstract}

\section{HARDWARE}

$\mathrm{I}_{\mathrm{c}}^{\mathrm{n}}$ all current brain-machine interface devices for both clinical and research applications, each electrode is independently connected to separate control systems. Examples of such devices include penetrating microelectrode arrays and cortical surface electrode arrays, and systems such as deep brain stimulators, and epilepsy treatment devices. These individually wired electrodes limit both the number and configuration of the electrodes that can be used to sample and stimulate tissues. Active circuits to reduce this wiring burden are limited by the mismatch between the rigid, planar nature of conventional, silicon electronics and the irregularly shaped tissue surfaces. Flexible electronics that are capable of intimate, noninvasive integration with the soft, curvilinear surfaces of the brain offer important opportunities for diagnosing and treating disease and for improving brain-machine interfaces. Here, we report new dense arrays of multiplexed electrodes using flexible electronics that can enable an unprecedented level of spatial and temporal electrocorticographic (ECoG) resolution over large areas of cortex. The extreme flexibility of the devices can further enable simultaneous sampling of gyral and intrasulcal ECoG to sample regions of the brain that were previously inaccessible or difficult to reach, but are known to carry enormously important information.

We demonstrate this technology in a sensor system

Manuscript received July 9, 2010. This work was supported in part by NIH NINDS grants RO1-NS041811-04 and RO1-NS48598-01, the Klingenstein Foundation, the Dana Foundation and the Epilepsy Research Foundation.

Authors J.V., J.B. and B.L. are with the Department of Bioengineering at the University of Pennsylvania, Philadelphia, PA 19104 USA.

Author B.L. is also with the Department of Neurology, Hospital of the University of Pennsylvania, Philadelphia, PA 19104 USA. (e-mail: littb@mail.med.upenn.edu). composed of 2016 silicon nanomembrane transistors configured to record electrical activity directly from the feline brain in vivo. The device samples with simultaneous submillimeter and submillisecond resolution through 288 amplified and multiplexed channels, requiring only 36 external wire connections. The design can be scaled up to much larger sizes, without dramatically increasing the number of external connections. We use this system to map visual and stimulation evoked potentials at high resolution, on the surface of primary visual cortex. This demonstration is one example of many possible uses of this technology in a new generation of minimally invasive clinical and research devices for medical applications, including cardiac electrophysiology and brain-computer interfaces $[1,2]$.

Interpretation of high bandwidth signals that are transduced from the above devices in brain require fast and accurate algorithms capable of mining large volumes of data to detect neurophysiological biomarkers without human intervention. One important set of biomarkers recently associated with epilepsy is high frequency oscillations

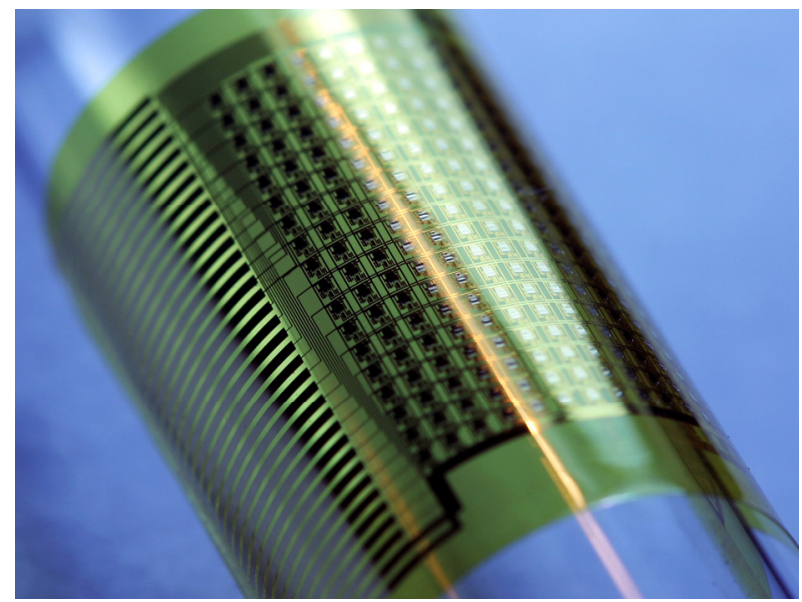

Fig. 1. 288-channel multiplexed, high-density active electrode using flexible silicon electronics for recording neural activity.

(HFOs).

\section{SOFTWARE}

High frequency oscillations (HFOs) have been observed in animal and human intracranial recordings during both normal and aberrant brain states. It has been proposed that the relationship between subclasses of these oscillations can be used to identify epileptic brain. Studies of HFOs in epilepsy have been hampered by selection bias arising 
primarily out of the need to reduce the volume of data so that clinicians can manually review it. We introduce an algorithm for detecting and classifying these signals automatically, and demonstrate the tractability of analyzing a data set of unprecedented size, over 31,000 channel-hours of intracranial electroencephalographic recordings (iEEG) from micro and macroelectrodes in humans. Using an unsupervised approach that does not presuppose a specific number of clusters in the data, we show direct evidence for the existence of distinct classes of transient oscillations within the 100 to $500 \mathrm{~Hz}$ frequency range in a population of nine neocortical epilepsy patients and two controls. The number of classes we find, four - three plus one putative artifact class - is consistent with prior studies that identify "ripple" and "fast ripple" oscillations using human-intensive methods, and additionally identifies a less examined class of mixed-frequency events [3].

\section{REFERENCES}

[1] Viventi J, Kim DH, Moss J, Kim Y-S, Blanco J, Anetta N, Hicks A, Xiao J, Huang Y, Callans DJ, Rogers JA, and Litt B. "A Conformal, Bio-interfaced Class of Silicon Electronics for Mapping Cardiac Electrophysiology," Science Translational Medicine, 2010 Mar 24;2(24).

[2] Kim DH, Viventi J, Amsden J, Xiao J, Vigeland L, Kim Y-S, Blanco J, Frechette E, Contreras D, Kaplan D, Omenetto F, Huang Y, Hwang K-C, Zakin M, Litt B, and Rogers JA. "Dissolvable Films of Silk Fibroin for Ultrathin, Conformal Bio-Integrated Electronics," Nature Materials, 2010 Apr 18.

[3] J. Blanco, "Unsupervised classification of high-frequency oscillations in neocortical epilepsy and control patients," Ph.D. dissertation, Dept. Bio. Eng., Univ. of Pennsylvania, Philadelphia, PA, 2010. 